\section{Stenting for obstructive iliac vein lesions}

\author{
Arjun Jayaraj, Seshadri Raju \\ The RANE Center for Venous \& \\ Lymphatic Diseases, St. Dominic \\ Hospital, Jackson, MS, USA
}

\begin{abstract}
Endovenous stenting has supplanted open surgery as the treatment of choice for iliocaval obstruction. This review provides a brief overview of such obstructive pathology and an in-depth assessment of femoroilio-caval stenting.
\end{abstract}

\section{Introduction}

Obstructive iliac vein lesions can result from primary or non-thrombotic and secondary or post-thrombotic pathology. The latter is responsible for a majority of all obstructive iliac vein lesions because of post thrombotic syndrome (PTS) development in 20 to $50 \%$ of patients who present with lower extremity deep venous thrombosis (DVT). ${ }^{1}$ Among non-thrombotic iliac vein lesions (NIVL), May-Thurner syndrome (MTS)/iliac vein compression syndrome (IVCS) is the most frequent etiology. ${ }^{2,3}$ While obstruction is seen primarily of the left common iliac vein, the right common iliac or either external iliac vein can also be involved in MTS/IVCS. Other less common causes of NIVL nclude retroperitoneal fibrosis; iatrogenic, blunt and penetrating trauma; congenital venous anomalies or benign/malignant tumors. Endovenous interventions have supplanted open surgery as the treatment of choice in patients with non-malignant obstructive iliac vein lesions. For patients with malignancy, venous stenting is used only when excision of the tumor/open reconstructive surgery is not possible and the goal of therapy is palliation. Adjunctive procedures such as femoral vein endophlebectomy/creation of arteriovenous fistula are added to venous stenting to provide improved inflow.

\section{Diagnosis}

Clinical manifestations of chronic venous insufficiency arising from obstructive lesions of the femoro-ilio-caval tract include swelling; varicosity; hyperpigmentation; venous claudication (pelvic, thigh or hip pain that develops after exercise); skin and subcutaneous inflammatory changes (eczema, lipodermatosclerosis, induration and venous ulcerations) of the affected limb. Of these, swelling has been noted to be the most common symptom followed by venous claudication in one review. ${ }^{4}$ In this study the incidence of active ulcers was approximately 19\%. Diagnostic testing is focused on confirming the presence of an obstructive femoro-ilio-caval lesion and determining etiology. Additionally, inflow and outflow patterns should be ascertained. Such testing includes venous duplex scanning, which should be used as a screening tool and performed in all patients with clinical presentation suggestive of obstructive venous pathology. Duplex scanning helps define location, etiology (obstructive $v s$ obstructive with concomitant valvular incompetence), and severity of the underlying problem. Air plethysmography can help evaluate global leg hemodynamics by measuring obstruction, reflux and calf pump function. Cross-sectional imaging including magnetic resonance (MR) venography and computed tomographic (CT) venography will identify obstructive pathology and provide sufficient information in most patients with regard to venous anatomy, collateral circulation and occlusion/stenosis. Ascending venography helps defines the site(s) of obstruction, collateral venous circulation and patterns of preferential flow. This is done by cannulation of the dorsal vein of the foot to assess the veins of the leg and through separate access of the common femoral vein to assess the ilio-caval system. Ambulatory venous pressure measurement by venous cannulation in the foot helps quantify venous hypertension. Intravascular ultrasound (IVUS) is used to assess degree of iliac vein stenosis before stenting; to assess apposition of stent to vein wall post-stenting and to evaluate stent/flow status during follow-up of patients with recurrent symptoms. IVUS is generally considered the gold standard from a diagnostic and therapeutic standpoint.

\section{Treatment}

\section{Conservative management}

Initial management of femoro-iliocaval obstruction is usually conservative, including frequent leg elevation, use of graduated compression stockings (30 to 40 $\mathrm{mm} \mathrm{Hg}$, and local wound care. Compression garments require strict compliance, which can be an issue due to a variety of reasons, including warm weather and a sense of tightness of the limb. Benefits attributed to graduated compression stock-
Correspondence: Arjun Jayaraj, The RANE Center, 971 Lakeland Drive, Suite \#401, Jackson, MS 39216, USA.

Tel.: +1.601.939.4230.

E-mail: arjunjayaraj2015@gmail.com

Key words: Iliac vein stenting; venous stents; obstructive iliac vein lesions; May Thurner syndrome; iliac vein compression syndrome.

Received for publication: 18 June 2017. Revision received: 14 August 2017.

Accepted for publication: 14 August 2017.

This work is licensed under a Creative Commons Attribution 4.0 License (by-nc 4.0).

CC Copyright A. Jayaraj and S. Raju, 2017

Licensee PAGEPress, Italy

Veins and Lymphatics 2017; 6:6855

doi:10.4081/vl.2017.6855

ings arise from their ability to impact venous hemodynamics, skin circulation, and calf muscle pump function. Patients with persistent disabling symptoms not responding to conservative measures should be considered for endovascular intervention.

\section{Endovascular treatment}

Femoro-ilio-caval stenting has become the primary treatment for obstructive venous pathology. Open approach is reserved for patients who are not candidates for or who have failed an endovascular approach. Endovascular intervention is usually performed under general anesthesia (some interventionalists prefer moderate intravenous sedation) given the frequent severe intraoperative pain/discomfort associated with balloon angioplasty. Access to the mid-thigh femoral vein is obtained under ultrasound guidance. This allows angioplasty/stenting of the common femoral vein if needed, without being impeded by the sheath. A 0.035 Glide wire (Terumo Medical Corp, Somerset, NJ) is passed into the inferior vena cava and a short $(10 \mathrm{~cm}) 11 \mathrm{Fr}$ sheath is placed. An ascending venogram of the ilio-femoral segments and inferior vena cava is performed. In patients with renal dysfunction, the venogram is skipped. Intravascular ultrasound [Volcano, San Diego, CA] is then performed using the 0.035 " catheter and planimetric measurements of the luminal areas of the common femoral vein (CFV), external iliac vein (EIV) and common iliac vein (CIV) are made. $125 \mathrm{~mm}^{2}$, $150 \mathrm{~mm}^{2}$ and $200 \mathrm{~mm}^{2}$ are used as normal luminal area cutoffs in the CFV, EIV and CIV respectively. Any decrease in luminal 
areas below the reference values shown in Table 1 in a symptomatic patient is considered abnormal, meriting angioplasty and stenting. ${ }^{5}$

It is important avoid using any particular threshold value of stenosis for treatment because unlike the arterial system, elevated peripheral venous pressure is the driving force behind clinical symptoms/signs. In this regard, even a minor stenosis of around $30 \%$ or so can significantly elevate venous pressure in postthrombotic limbs. The basis of CVI is venous hypertension and the aim of stenting is to relieve the same. Predilation of the stenosis is performed using an $18 \times 60 \mathrm{~mm}$ Atlas angioplasty balloon (Bard Peripheral Vascular, Tempe, AZ). Stenting is then carried out using 18$20 \mathrm{~mm}$ Wallstents (Boston Scientific, Marlborough, MA) with landing zones determined by IVUS - defined bony landmarks. The proximal landing zone is typically $1-2 \mathrm{~cm}$ above the iliac confluence that can be related to the corresponding vertebral body (upper, middle or lower border). The distal landing zone is an area of adequate inflow in the CFV and can be related to a body landmark of the pubic ramus, femoral head or lesser trochanter. Attention must be paid to the vein at the level of the inguinal ligament since this is often an area of compression. Stenting across the inguinal ligament must be performed in these cases and can be done with good results (Figure 1). ${ }^{6}$ Given the relatively low radial strength of the Wallstent, a Gianturco Z stent (Cook Medical, Bloomington, Indiana) is used to provide additional strength across the confluence with an extension of the Gianturco stent beyond the Wallstent proximally into the IVC. The Gianturco Z stent should be oversized relative to the Wallstent with an overlap of the lower half of the $\mathrm{Z}$ stent within the Wallstent to prevent stent embolization. An overlap of $3 \mathrm{~cm}$ or so between each Wallstent in the stack is required to compensate for foreshortening during post-dilation. Post-dilation is performed using the $18 \times 60 \mathrm{~mm}$ angioplasty balloon. Completion IVUS is performed to ensure adequacy of the luminal area. Any residual narrowing on IVUS interrogation is overcome by repeat dilation using sustained inflation or, if necessary, a larger caliber angioplasty balloon $(20 \mathrm{~mm})$. Completion venogram is then performed. The $11 \mathrm{Fr}$ sheath is subsequently withdrawn to just outside the vein and a Surgicel Fibrillar patch (Ethicon, Somerville, NJ) is introduced via the sheath to aid in local hemostasis. Manual pressure is maintained to complement the hemostatic effect. A retrograde access through the right internal jugular vein is also used if the obstruction cannot be crossed from the femoral access. Kurlinsky at al. reported their experience with stenting 91 post-thrombotic iliac or iliofemoral veins. Primary, primary assisted and secondary patencies at 3 years were $71 \%, 90 \%$ and $95 \%$, respectively. ${ }^{7}$ In the largest single institutional study, 6-year follow-up data of 982 stents placed for chronic nonmalignant obstructive lesions of the femoro-ilio-caval vein demonstrated primary, primary assisted and secondary patency rates of $79 \%$, $100 \%$, and $100 \%$ in nonthrombotic disease and $57 \%, 80 \%$, and $86 \%$ in post-thrombotic disease, respectively. ${ }^{8}$

\section{Chronic total occlusion}

Recanalization of chronic total occlusion (CTO) is most commonly done through the use of an 0.035" Glidecath (Terumo Medical Corp, Somerset, NJ) and 0.035 " Glidewire. A mid-thigh femoral vein approach is satisfactory in most instances with a short entry to lesion length, allowing greater pushability of instruments. Access of the profunda femoris vein or the popliteal vein may sometimes be required depending on inflow. The internal jugular vein approach is sometimes necessary when the antegrade approach fails. A body floss tech-

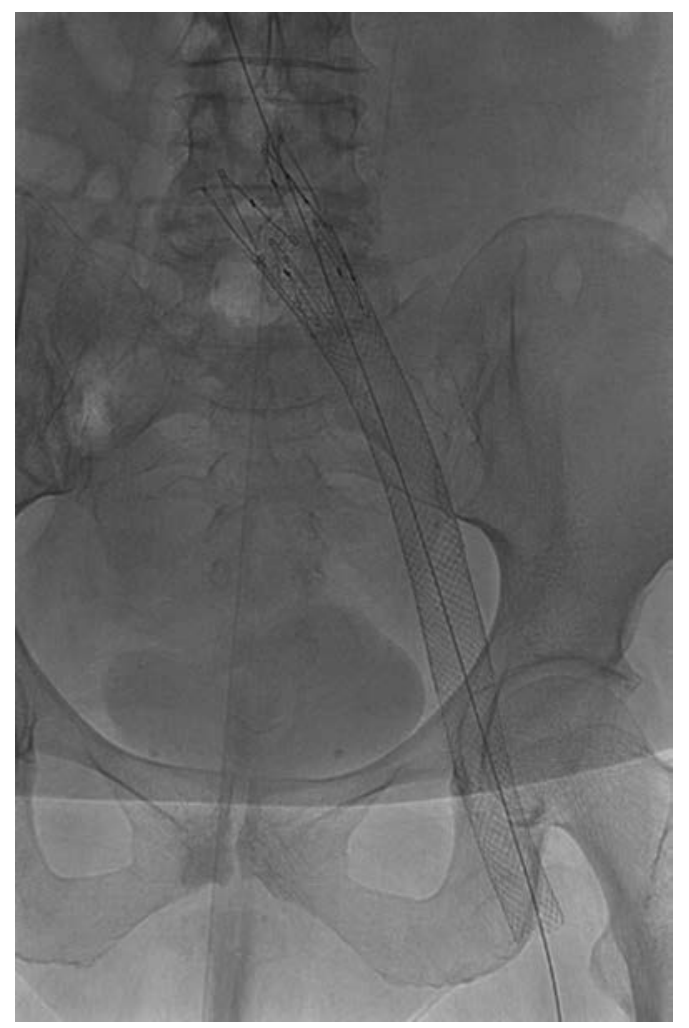

Figure 1. Stenting across the inguinal ligament using Gianturco $\mathrm{Z}$ stent/Wallstent combination. Stenting can be carried out safely across the inguinal ligament without compromising stent outcomes.
Table 1. Luminal area values.

\begin{tabular}{lcc} 
Vein & $\begin{array}{c}\text { Luminal area } \\
\left(\mathrm{mm}^{2}\right)\end{array}$ & $\begin{array}{c}\text { Diameter } \\
(\mathrm{mm})\end{array}$ \\
CFV & 125 & 12 \\
EIV & 150 & 14 \\
\hline CIV & 200 & 16 \\
\hline
\end{tabular}

$\mathrm{CFV}$, common femoral vein; EIV, external iliac vein; CIV, common

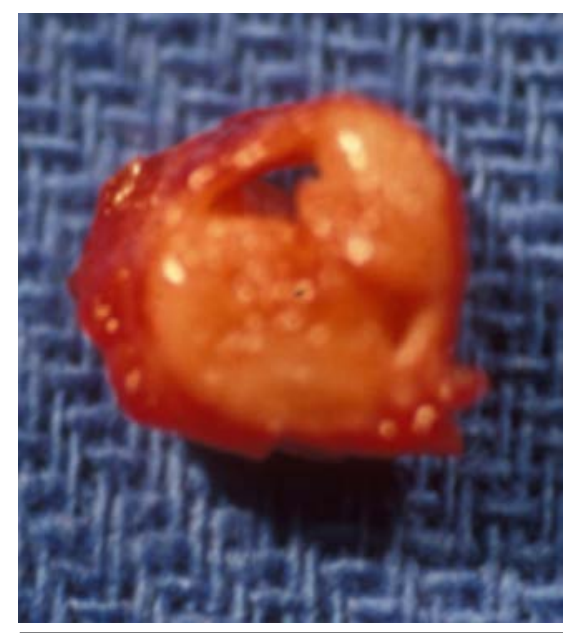

Figure 2. Trabeculae in chronically thrombosed vein segment. Successful recanalization requires localization and subsequent threading of guidewire through such trabeculae. 
nique may be necessary occasionally as described by Kolbel and colleagues. ${ }^{9}$ Less commonly, other devices may be needed for recanalization of CTO lesions including Quick-Cross support catheter (Spectranetics Corp, Colorado Springs, CO) and the TriForce peripheral crossing set (Cook Medical, Bloomington, IN). The key is to gain entry into a trabecular channel, which then leads one all the way into a patent segment (Figure 2). There is no role for subintimal entry/angioplasty as is utilized to perform recanalization of arterial segment CTOs.

Once wire recanalization is attained, angioplasty is performed as previously outlined. Sometimes, angioplasty with sequentially larger balloons may have to be employed due to inadequate purchase of the initial large caliber balloon. Rupture/hemorrhage from this maneuver is extremely rare. Angioplasty is carried out caudal to cranial (femoral access) or cranial to caudal (jugular access) as this facilitates easier retrieval of the angioplasty balloon should it disrupt. The likelihood of the latter happening is higher in CTO than in stenotic lesions. Use of stents, post-dilation, IVUS interrogation and venogram are all performed as previously described. Fatima et al reported $90 \%$ two-year patency rate and $80 \%$ symptomfree survival in a series of 28 patients undergoing inferior vena cava (IVC) stenting for occlusion (16)/high grade stenosis. ${ }^{12}$ Freedom from reintervention in this group, which included 13 patients with IVC filters at the 2-year mark, was $84 \% .^{10}$ Raju et al described their experience in 120 patients with chronic obstruction of the IVC and reported cumulative stent patency of $82 \%$ at 2 years. ${ }^{11}$ Relief of pain and swelling of $74 \%$ and $51 \%$ respectively was noted at 42 months follow-up. The cumulative rate of complete ulcer healing in this group at 2 years was $63 \% .{ }^{11}$ More recently Murphy et al published outcomes following endovascular management of CTO of the inferior vena cava/iliac veins and noted primary, primary assisted, and secondary patency rates at 60 months of $52 \%, 85 \%$, and $93 \%$, respectively. The authors reported complete cumulative relief of pain and swelling in $66 \%$ and $41 \%$ respectively, with the venous clinical severity score improving from $8.4 \pm 5.1$ (range, 4-27) prior to intervention to $3.9 \pm 3.2$ (range, 0-14) post-intervention $(\mathrm{P}<0.001) .{ }^{12}$

\section{Stenting across inferior vena cava filters}

Inferior vena cava filters can over time serve as a nidus for a fibrotic reaction that leads to IVC stenosis/occlusion. Trapped emboli may start the process in some instances. The occluded filter and IVC segment must be recanalized to provide adequate outflow. This can be accomplished by removal of the filter if possible, or crushing the filter and stenting across it. $24 \mathrm{~mm}$ Wallstents are typically used in the IVC/across IVC filters and have had good results (Figure 3A-F).

Patients need to be counseled about loss of filter protection for pulmonary embolism consequent to such procedures. In a review of $121 \mathrm{limbs}$ that underwent stenting for postthrombotic ilio-caval occlusions, limbs stented for recanalized occlusions with $(n=23)$ and without IVC filters $(n=92)$ showed no difference in patency rates. Cumulative primary and secondary patency rates were $30 \%$ and $35 \%(\mathrm{P}=0.97)$ and $71 \%$ and $73 \%(\mathrm{P}=0.93)$ respectively. The authors conclude that the primary factor affecting stent patency in such patients was severity of postthrombotic disease and not presence of a filter. ${ }^{13}$

\section{Bilateral iliocaval stenting}

There is a limited role for simultaneous bilateral femoro-ilio-caval stenting, except for bilateral recanalization procedures. Typically, the more affected leg is stented, giving adequate time for the less affected leg to improve from off-loading of cross collaterals. In patients with persistent symptoms in the contralateral lower extremity, contralateral stenting can be pursued. In the presence of a prior Gianturco Z stent, the flowering technique is used wherein the cranial nylon suture of the new Gianturco Z stent is cut so as to allow the struts to flower out and allow it to mesh with the older contralateral Gianturco Z stent (Figure 4).

If the contralateral stent is a Wallstent then a fenestrum needs to be created by wire access across the wall stent interstice and dilation of same using an 18x60 mm angioplasty balloon. Stenting across this fenestrum is accomplished using a combination of Wallstent and Gianturco 'crown' as previously described. The wide struts of the Gianturco $\mathrm{Z}$ stent lining the fenestrum allow free flow in the contralateral stent across the fenestrum (Figure 5).

The authors' preference is to use a bilateral Wallstent/Gianturco Z stent combination for management of the iliac confluence as opposed to the apposition, double barrel or fenestrum techniques. Raju et al reported 2-year cumulative primary and secondary patency of $69 \%$ and $93 \%$ respectively in 273 limbs intervened on by using the $\mathrm{Z}$ stent technique. $^{14}$

\section{Hybrid treatment}

Venous stents are combined with femoral vein endophlebectomy/patch angioplasty or arteriovenous fistula (AVF) creation or both to improve inflow into the stents. The stent is typically placed proximal to the patch or can be extended into the patch. The common femoral or superficial femoral artery can be used for inflow to create the fistula, which is typically fashioned using a 4 to $6 \mathrm{~mm}$ externally supported PTFE graft. Acceptable outcomes following endophlebectomy and AVF creation have been reported. ${ }^{15-18}$

\section{Anticoagulation}

For perioperative thromboprophylaxis, the authors use enoxaparin $40 \mathrm{mg}$ given subcutaneously preoperatively, in addition to bivalrudin $75 \mathrm{mg}$ given intravenously in the operating room prior to the start of the procedure. The authors use this combination of direct and indirect thrombin inhibitors to reduce early stent complications including thrombosis. Following iliocaval stenting, in patients with thrombophilia/post-thrombotic syndrome therapeutic anticoagulation is continued. Those in whom thrombophilia workup is negative and who have non-thrombotic femoro-iliocaval lesion, aspirin $81 \mathrm{mg}$ with cilastazol $50 \mathrm{mg}$ twice daily is typically used unless contraindicated. The latter is used for its suppressive effect on neointimal hyperplasia. Presence of significant in-stent restenosis, but with no symptom recurrence is an indication to switch to apixaban $2.5 \mathrm{mg}$ on a twice-daily basis. Recurrence of symptoms is an indication for repeat IVUS interrogation and possible angioplasty.

\section{Follow-up}

Venous duplex ultrasound is performed on post-operative day one to obtain baseline post-procedure metrics, including stent patency, and to assess stent compression and/or in-stent restenosis. These parameters are again evaluated by repeat duplex ultrasound in addition to assessing symptom relief at clinic visits 2 weeks and 4 weeks post-procedure. Three to six monthly follow-up is required subsequently, which is gradually reduced to annual follow up depending on symptoms and status of stents. More frequent follow-up is typically required for PTS and recanalization patients. 

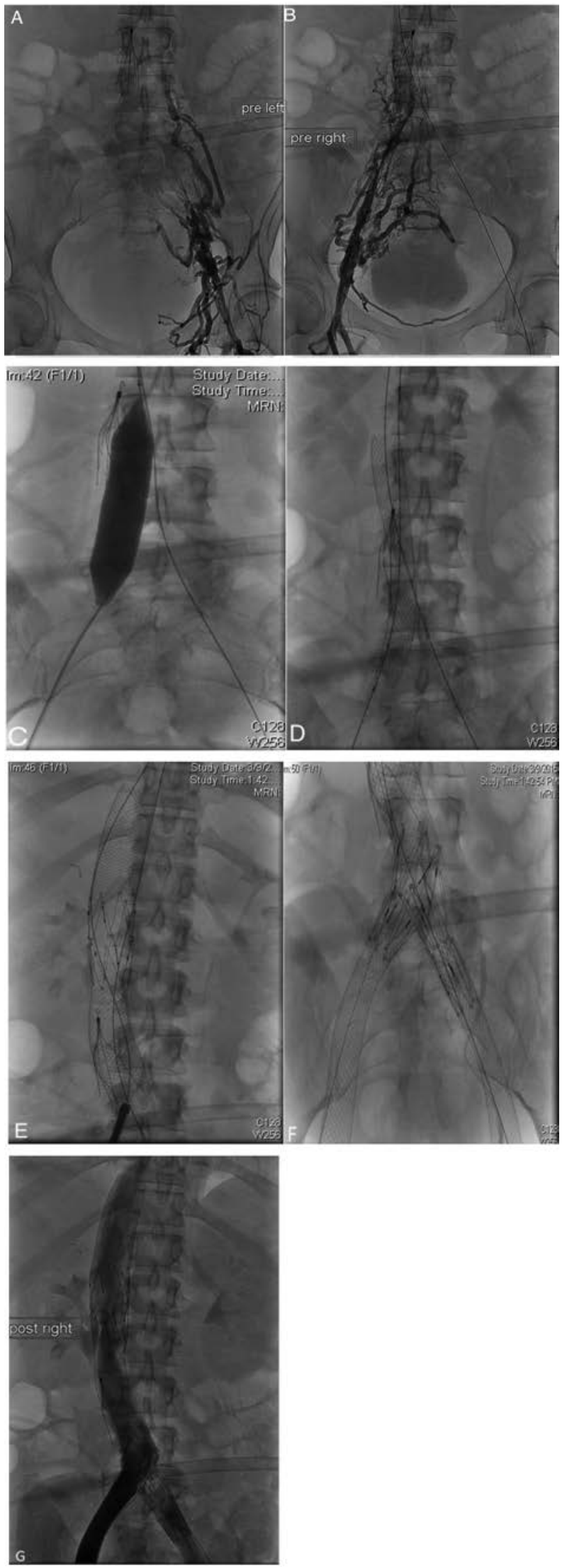

Figure 3. A-G) Recanalization - Iliocaval occlusion with IVC filter (A and B: right and left venogram demonstrating bilateral CTO with occluded IVC filter; C and D: IVC filter crush with $24 \mathrm{~mm}$ angioplasty balloon and subsequent stenting across filter; E: Stenting up to hepatic vein confluence to provide good outflow using Gianturco $Z$ stent across renal veins; F: Iliocaval confluence stenting using Gianturco Z stent/Wall stent technique; G: completion venogram). 


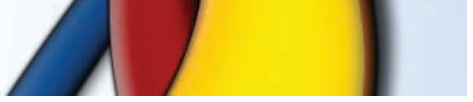

\section{Complications of iliac stenting and their management}

Femoro-ilio-caval stenting can be performed with minimal mortality and low morbidity. Reintervention is required at times. Raju et al. noted a reintervention rate of $13 \%$ following femoro-ilio-caval stenting in 1085 limbs. Median time to reintervention after the initial procedure was 15 months. Post-reintervention the group reported cumulative improvement in pain and swelling of $67 \%$ and $72 \%$, respectively, at 18 months follow-up. Complete cumulative healing of venous dermatitis/ulcer at 12 months' post-reintervention was $90 \%{ }^{19}$ Some of the potential complications that can be encountered are considered in the following paragraphs.

\section{Access site-related}

Such complications can be reduced by use of ultrasound guidance for access. Clinically significant hematoma/arterial injury/pseudoaneurysm/nerve injury is extremely rare $(<0.1 \%)$. In most cases access site hematomas can be effectively managed with compression and delayed start of anticoagulation. Pseudoaneurysm, when indicated, is best managed by ultrasound-guided thrombin injection. Patients who sustain nerve injuries attain symptom relief over time. However, counseling and setting realistic expectations are key.

\section{Vein injury/rupture}

Frank rupture of the intervened vein is uncommon due to relatively low pressure and significant periadventitial fibrosis around the vein. When rupture occurs use of a stent graft can help exclude the site of extravasation. ${ }^{20}$

\section{In-stent restenosis}

In-stent restenosis (ISR) arises from either thrombus buildup or neointimal hyperplasia within the stent. The authors note an incidence of up to $25 \%$ in their experience. Patients presenting with recurrence of symptoms require reintervention with angioplasty. This often involves use of an angioplasty balloon larger than the rated size of the stent used (e.g., for a $20 \mathrm{~mm}$ stent we can use a $22 \mathrm{~mm}$ angioplasty balloon). This is termed hyperdilation as opposed to isodilation, which is dilation with use of an angioplasty balloon of the same rated diameter as the stent. Therapeutic anticoagulation and use of cilastazol immediately after index stent procedure may help reduce occurrence.

\section{Stent compression}

Stent compression occurs due to extrinsic compression of the stent due to fibrotic tissue build up. This is a phenomenon unique to the venous system. Incidence is significantly lower than ISR, with treatment being hyperdilation in symptomatic patients. Larger caliber balloons may have to be used to overcome the stent compression (22-24 mm) than in ISR.

\section{Stent thrombosis}

Layering of thrombus within the stent occurs can occur due to poor inflow, poor outflow or mechanical effects of an inadequate stent stack with potential for stent thrombosis. The overall incidence of stent thrombosis is approximately $3.5 \%$ in the authors experience, with chronic thrombosis more common than acute. ${ }^{21}$ Contributing factors to an inadequate stent stack include use of undersized stent and understenting (not covering all areas of disease). Lack of perioperative use of anticoagulation/ antiplatelet agent(s) can also contribute to stent thrombosis. Restenting after fracturing the previously placed undersized stents with large caliber angioplasty balloons or extension of stent stack proximally or distally as the case may be is required for undersized stent and under stenting, respectively. For acute/subacute occlusions treatment is with pharmacomechanical thrombectomy +/balloon maceration (no pulmonary embolisms in the authors experience). For more chronic occlusions (CTO), recanalization can be pursued as described earlier. Acceptable results have been noted in both situations. Laser recanalization or radiofrequency wire recanalization has also been used as a last resort in occluded stents with modest success.

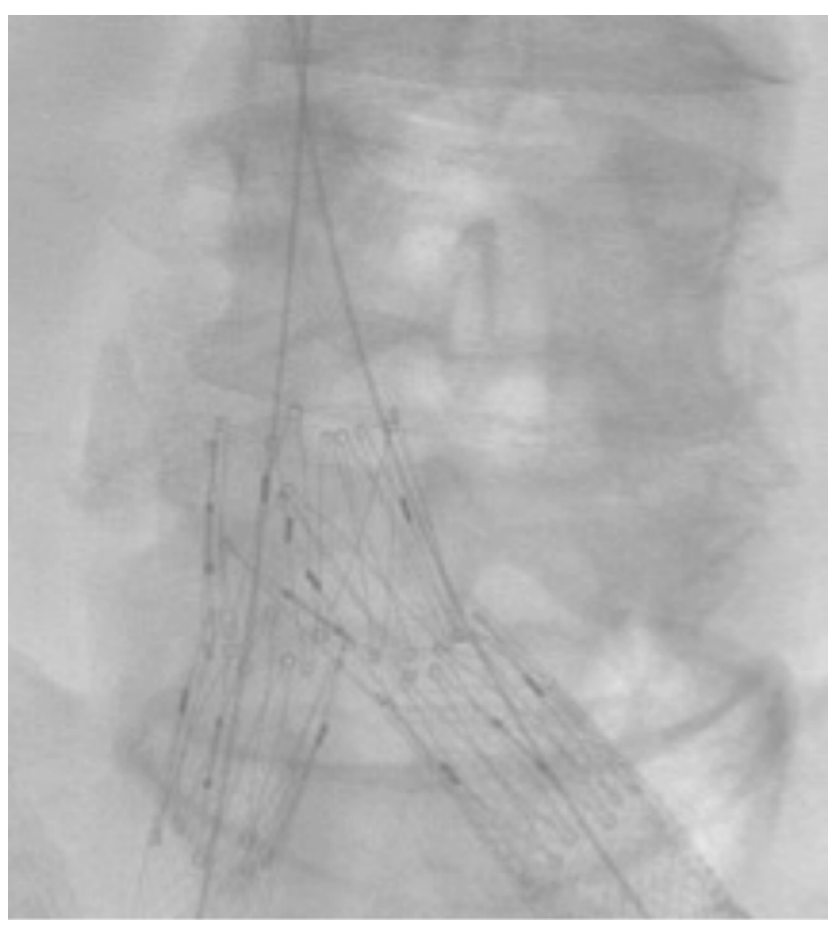

Figure 4. Bilateral stenting with use of Gianturco $\mathrm{Z}$ stents overcomes challenges arising from double barrel and fenestral techniques.

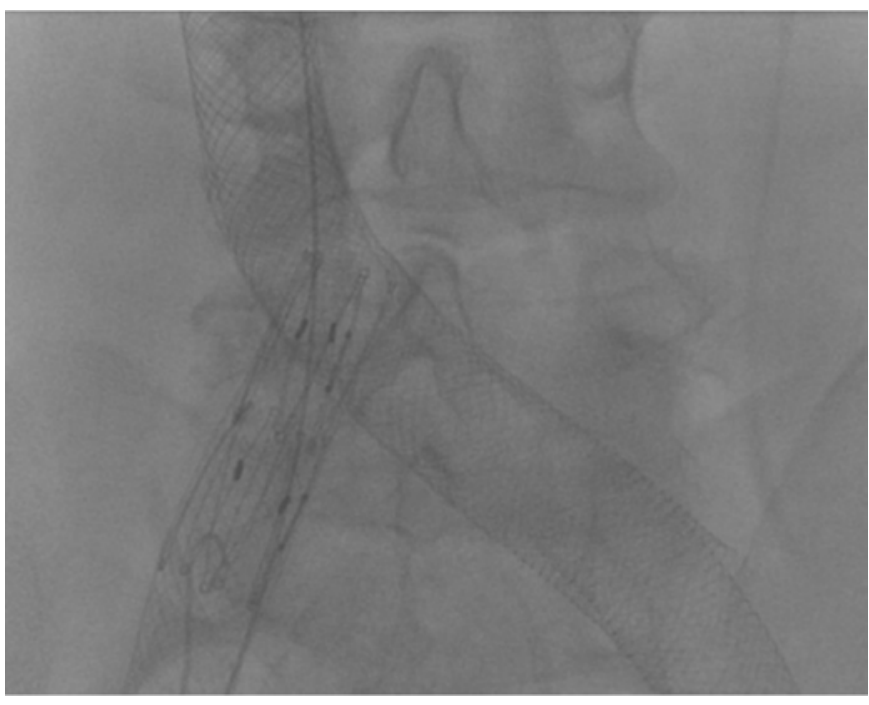

Figure 5. Bilateral stenting using fenestrum creation through contralateral stent. Used when contralateral stent extends to ipsilateral caval wall and "standard" Gianturco Z stent/Wallstent combination is not an option. 


\section{Contralateral iliac vein thrombosis}

This is a rare event occurring from jailing of contralateral common iliac vein by ipsilateral stent. It can be overcome by use of Wallstent-Gianturco Z stent (Boston Scientific, Marlborough, MA- Cook Medical, Bloomington, IN) combination and limiting extension into IVC. The latter technique reduces the risk of contralateral thrombosis by up to $85 \% .^{22}$

\section{Stent migration}

Occurs due to the choke point effect of the iliac confluence. It is imperative to extend the stent stack proximal to the confluence to overcome this effect. Furthermore, use of the Gianturco $\mathrm{Z}$ stent (Cook Medical, Bloomington, IN) helps provide additional radial force and checks migration.

\section{Mortality}

Worldwide experience has proven venous stenting to be a low-risk procedure with negligible morbidity and mortality. ${ }^{23}$

\section{References}

1. Kahn SR, Comerota AJ, Cushman M, et al. The postthrombotic syndrome: evidence-based prevention, diagnosis, and treatment strategies: a scientific statement from the American Heart Association. Circulation 2014;130: 1636-61.

2. Raju S, Neglen P. High prevalence of nonthrombotic iliac vein lesions in chronic venous disease: a permissive role in pathogenicity. J Vasc Surg 2006;44:136-43; discussion 44.

3. Titus JM, Moise MA, Bena J, et al. Iliofemoral stenting for venous occlusive disease. J Vasc Surg 2011;53:70612.

4. Garg N, Gloviczki P, Karimi KM, et al.
Factors affecting outcome of open and hybrid reconstructions for nonmalignant obstruction of iliofemoral veins and inferior vena cava. J Vasc Surg 2011;53:383-93.

5. Raju S BW, Crim W, Jayaraj A. Optimal sizing of iliac vein stents. Phlebol Venous Forum R Soc Med 2017 [In press].

6. Neglen P, Tackett TP, Jr., Raju S. Venous stenting across the inguinal ligament. J Vasc Surg 2008;48:1255-61.

7. Kurklinsky AK, Bjarnason H, Friese JL, et al. Outcomes of venoplasty with stent placement for chronic thrombosis of the iliac and femoral veins: single-center experience. J Vasc Interv Radiol 2012;23:1009-15.

8. Neglen P, Hollis KC, Olivier J, Raju S. Stenting of the venous outflow in chronic venous disease: long-term stent-related outcome, clinical, and hemodynamic result. J Vasc Surg 2007;46:979-90.

9. Kolbel T, Lindh M, Akesson M, et al. Chronic iliac vein occlusion: midterm results of endovascular recanalization. J Endovase Ther 2009;16:483-91.

10. Fatima J, AlGaby A, Bena J, et al. Technical considerations, outcomes, and durability of inferior vena cava stenting. J Vasc Surg 2015;3:380-8.

11. Raju S, Hollis K, Neglen P. Obstructive lesions of the inferior vena cava: clinical features and endovenous treatment. J Vasc Surg 2006;44:820-7.

12. Murphy EH, Johns B, Varney E, Raju S. Endovascular management of chronic total occlusions of the inferior vena cava and iliac veins. J Vasc Surg Venous Lymph Disord 2017;5:47-59.

13. Neglen P, Oglesbee M, Olivier J, Raju S. Stenting of chronically obstructed inferior vena cava filters. J Vasc Surg 2011;54:153-61.

14. Raju S, Ward M, Jr., Kirk O. A modification of iliac vein stent technique. Ann
Vasc Surg 2014;28:1485-92.

15. de Wolf MA, Jalaie H, van Laanen JH, et al. Endophlebectomy of the common femoral vein and arteriovenous fistula creation as adjuncts to venous stenting for post-thrombotic syndrome. $\mathrm{Br} \mathrm{J}$ Surg 2017;104:718-25.

16. de Wolf MA, Arnoldussen CW, Wittens $\mathrm{CH}$. Indications for endophlebectomy and/or arteriovenous fistula after stenting. Phlebol Venous Forum R Soc Med 2013;28 Suppl 1:123-8.

17. Comerota AJ. Venous thrombectomy and arteriovenous fistula versus anticoagulation in the treatment of iliofemoral venous thrombosis. J Vasc Surg 1992;15:887-9.

18. Comerota AJ, Grewal NK, Thakur S, Assi Z. Endovenectomy of the common femoral vein and intraoperative iliac vein recanalization for chronic iliofemoral venous occlusion. J Vasc Surg 2010;52:243-7.

19. Raju S, Tackett P, Jr., Neglen P. Reinterventions for nonocclusive iliofemoral venous stent malfunctions. J Vasc Surg 2009;49:511-8.

20. Adams MK, Anaya-Ayala JE, Davies MG, Bismuth J, Peden EK. Endovascular management of iliac vein rupture during percutaneous interventions for occlusive lesions. Ann Vasc Surg 2012;26:575e5-9.

21. Jayaraj ACW, Murphy EH, Raju S. Occlusion following iliocaval stentingcharacteristics and outcomes. J Vasc Surg 2016;63:53S-4S.

22. Murphy EH, Johns B, Varney E, et al. Deep venous thrombosis associated with caval extension of iliac stents. J Vasc Surg Venous Lymph Disord 2017;5:8-17.

23. Raju S. Best management options for chronic iliac vein stenosis and occlusion. J Vasc Surg 2013;57:1163-9. 